\title{
Poisson's Spot at New Shapes of Damage of a Circular Disk
}

\author{
D. Dimić $\tilde{C}^{a}$ AND D. ARSENOVIĆc,* \\ ${ }^{a}$ Faculty of Natural Sciences and Mathematics, University of Niš, Niš, Serbia \\ ${ }^{b}$ Institute of Physics, University of Belgrade, Belgrade, Serbia
}

Received: 18.12.2017 \& Accepted: 18.05.2020

Doi: $10.12693 /$ APhysPolA.138.345

*e-mail: arsenovic@ipb.ac.rs

\begin{abstract}
Poisson's spot is the bright point in the centre of the geometrical shadow of a circular object, as a consequence of Fresnel's diffraction. In the literature, the relative intensity of Poisson's spot has been examined in the case of small deviations from the circular cross-section of the circular diffraction obstacle, simulating a surface roughness. It was shown that small amounts of surface roughness can completely remove Poisson's spot. In this paper, a specially adapted analytical-numerical method is presented to study this phenomenon. With this method, the influence of larger deviations from the circularity of a diffraction obstacle on Fresnel diffraction can be examined. The deviations from the ideal cross-section of a lightened circular object are of the semi-circular shape. The effect of different modifications to the cross-section of this type was analyzed. All of the simulated diffraction images are similar to the case of a circular cross-section, showing Poisson's spot. However, the obtained theoretical results show that the relative intensity of Poisson's spot falls oscillatorily as the size of deviations increases. The oscillatory dependence is explained by using the concept of Fresnel zones. The method can be used for modeling future diffraction experiments of both electro-magnetic and matter waves.
\end{abstract}

topics: interferometry, Poisson's spot, Fresnel zone

\section{Introduction}

Great possibilities of computer simulation in science enabled research in physics allowing to examine once again some physical phenomena. One of them, connected with the wave nature of light, is Poisson's spot which is a consequence of light diffraction in the shadow of a circular opaque disk $[1,2]$. The experimental confirmation of the existence of that spot led to winning of Fresnel's wave theory of light [3] over Newton's corpuscular theory [4]. However, at the beginning of this century, it was reported theoretically [5-9] that the electromagnetic energy (EME) flow lines can be observed as possible trajectories of photons. Based on this, in [8] authors simplified the Rayleigh-Sommerfeld integral $[10,11]$ and came up with an algorithm for determining a relative intensity dependence of Poisson's spot on the radius of the blocking disk, on the distance of the screen behind the disk, and on the wave length of monochromatic light, as well as an algorithm for drawing the EME flow lines as the possible trajectories of photons. At the same time, T. Reisinger et al. [12, 13] studied Poisson's spot by the diffraction of deuterium molecules and $C_{70}$ at both an ideal and rough disk. The authors assumed sinusoidal roughness and they performed appropriate simulations by using an algorithm devised by Dauger [14]. In the mentioned articles, the simulated surface roughness was relatively small.
In the present paper, we shall simulate the influence of relatively large deviations from the ideal circular cross-section of the circular diffraction object on the relative intensity of Poisson's spot. We would also like to refer to the authors addressing the same problem but with different applications and slightly different approaches [15-17].

We have developed an analytical-numerical method for studying the influence of larger deviations of a semi-circular shape on the relative intensity of the bright spot. The method introduced in Sect. 2 uses the Rayleigh-Sommerfeld integral and few new functions to estimate that influence. In Sect. 3, we shall examine three specific arrangements of larger deviations from the ideal circular cross-section. In the centre of the shadow of each of them, a bright spot appears. The relative intensities of the central bright spot show an oscillatory dependence on the size of disk damage. However, further on we show that in the case of a large number of small deviations of such a shape an oscillatory dependence disappears. The relative intensity of Poisson's spot falls continuously by increasing the size of the disk damage. Throughout Sect. 4, the diffraction of monochromatic light of wavelength $\lambda=500 \mathrm{~nm}$ incident on a rough disk with the radius of $R=0.5 \mathrm{~mm}$ was assumed. The roughness is simulated by 48 circles of the same radius with the centres on the edge of the circular disk. They cut equal parts from the simulated disk. The influence of such roughness on the relative 
intensity of Poisson's spot depends on the radius of the circles which cut the disk as well as on the distance $z$ of the disk to screen. In Sect. 5 , the results are analyzed and we indicate the possibility of further theoretical research which this method gives in the area of understanding the nature of light, as well as some practical applications.

\section{Description of the method}

Let us consider a two-dimensional opaque circular disk in the plane $z=0$ (Fig. 1). The centre of the circular opaque disk is $(x=0, y=0, z=0)$. We are interested in how a large circular deviation of such a disk influences the diffraction of waves. To simulate the deviation, one part from the circular disk is separated. In the simulation, the matter waves (here: the plane waves) fall on a rough disk in a half-plane of $z=0$. The light is described by the electric and magnetic field, namely

$$
\begin{aligned}
& \boldsymbol{E}(\boldsymbol{r}, t)=\operatorname{Re}\left\{\boldsymbol{E}(\boldsymbol{r}) \mathrm{e}^{-\mathrm{i} \omega t}\right\}, \\
& \boldsymbol{B}(\boldsymbol{r}, t)=\operatorname{Re}\left\{\boldsymbol{B}(\boldsymbol{r}) \mathrm{e}^{-\mathrm{i} \omega t}\right\},
\end{aligned}
$$

while particles with the mass are described by the wave function $\psi(\boldsymbol{r}, t)=\psi(\boldsymbol{r}) \mathrm{e}^{-\mathrm{i} \omega t}$, where $\omega$ is the angular frequency. For all the three quantities, $\boldsymbol{E}(\boldsymbol{r}), \boldsymbol{B}(\boldsymbol{r})$ and $\psi(\boldsymbol{r})$, the spatial part $A(\boldsymbol{r}) \in C$ satisfies the Hemholtz equation

$$
\nabla^{2} A(\boldsymbol{r})+k^{2} A(\boldsymbol{r})=0
$$

with the wave number $k=\frac{2 \pi}{\lambda}=\frac{\omega}{v}$, where $v$ is the velocity of the wave. This type of equation follows both from Maxwell's and Schrödinger's equations for a free particle. When solving (1), it is necessary to be in the area $z>0$, where the boundary condition is given as the value of the required function $A(\boldsymbol{r})$ in the plane $z=0$. We assume that for an incident wave the initial condition $A_{0}(x, y, z=0)$ is:

$$
A_{0}(x, y)= \begin{cases}A_{0}, & \text { for points }(x, y) \text { which } \\ & \text { the rough disk does not cover } \\ 0, & \text { for }(x, y) \in \text { the rough disk }\end{cases}
$$

For a situation when the wave amplitude $A(\boldsymbol{r})$ is constant in the plane $z=0$, the solution of (1) is

$$
\begin{gathered}
A(x, y, z)=-\frac{\mathrm{i}}{\lambda} \int_{S} A_{0}\left(x_{m}, y_{m}\right) \frac{\mathrm{e}^{\mathrm{i} k r}}{r} \\
\times\left(1-\frac{1}{\mathrm{i} k r}\right) \cos (\theta) \mathrm{d} x_{m} \mathrm{~d} y_{m},
\end{gathered}
$$

where

$$
\begin{aligned}
& r=\sqrt{\left(x-x_{m}\right)^{2}+\left(y-y_{m}\right)^{2}+z^{2}}, \\
& \cos (\theta)=\frac{z}{r}
\end{aligned}
$$

and where $S$ is the surface on which the initial condition is set by the function $A_{0}\left(x_{m}, y_{m}\right)$. In order to simplify the calculation, we apply Babinet's principle [18]. The field is defined as the difference between the field which would be created by a free

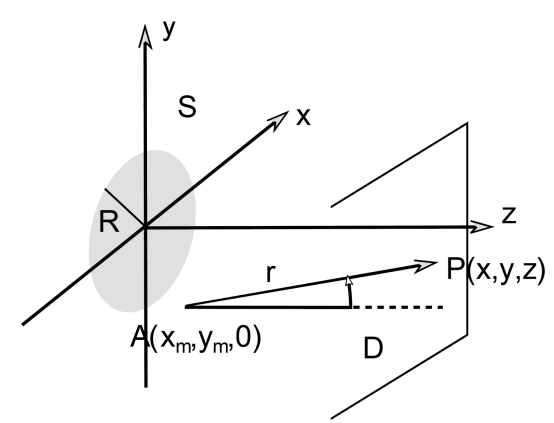

Fig. 1. The scheme of the experiment of the diffraction of the monochromatic laser light on the ideal circular opaque disk.

plane wave and the one created by the illuminated rough disk in $z=0$ plane. This is expressed as

$$
A(x, y, z)=A_{0} \mathrm{e}^{\mathrm{i} k z}-A_{h}(x, y, z) .
$$

Now, $A_{h}(x, y, z)$ is given in accordance with (3), only that $A(x, y, z=0)=A_{0}(x, y)$, i.e.,

$$
A_{0}(x, y)= \begin{cases}0, & \text { for }(x, y) \text { which } \\ & \text { the rough disk does not cover } \\ A_{0}, & \text { for }(x, y) \in \text { the rough disk }\end{cases}
$$

Thus,

$$
\begin{aligned}
& A_{h}(x, y, z)=-\frac{\mathrm{i} A_{0}}{\lambda} \int_{S_{h}} \frac{\mathrm{e}^{\mathrm{i} k r}}{r} \\
& \quad \times\left(1-\frac{1}{\mathrm{i} k r}\right) \cos (\theta) \mathrm{d} x_{m} \mathrm{~d} y_{m},
\end{aligned}
$$

where $S_{h}$ is the surface in the $z=0$ plane which the rough disk covers. It is suitable to use the cylindrical coordinates:

$$
\begin{aligned}
& x_{m}-x=\rho_{m} \cos \left(\varphi_{m}\right), \\
& y_{m}-y=\rho_{m} \sin \left(\varphi_{m}\right)
\end{aligned}
$$

where $r=\sqrt{\rho_{m}^{2}+z^{2}}$, and $\cos (\theta)=\frac{z}{r}$. Note that the integrand in (7) does not depend on $\varphi_{m}$. The expression can be then simplified as follows:

$$
A_{h}\left(\rho_{m}, \varphi_{m}, z\right)=I_{\rho_{m}}\left(I_{\varphi_{m}}\right) .
$$

The size:

$$
I_{\varphi_{m}}=\int_{S_{\varphi_{m}\left(\rho_{m}\right)}} \mathrm{d} \varphi_{m},
$$

is the total size of the angles under which the intervals of the circle $O_{m}$ and the rough disk cuttings are seen (Fig. 2). For a given set of $\rho_{m}$, the set $S_{\varphi_{m\left(\rho_{m}\right)}}$ of all values $\phi_{m}$ is obtained, so the point with coordinates $\left(z=0, \varphi_{m}, \rho_{m}\right)$ belongs to the unlighted part of the disk. In order to find the limits of the integration, firstly we will solve several geometrical tasks.

Let us observe two circles of the radius $R_{1}$ and $R_{2}$ in the distance $X>0$ (Fig. 3). For the simplicity of notes, we introduce a function:

$$
\operatorname{mfarccosx}= \begin{cases}0 & x>1 \\ \arccos (x) & -1 \leq x \leq 1 \\ \pi & x<-1\end{cases}
$$




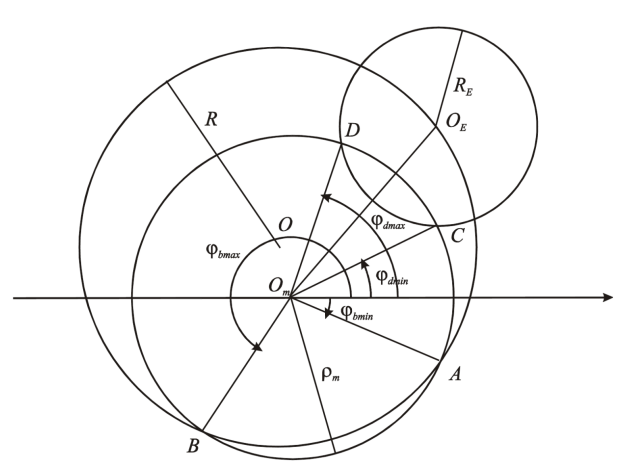

Fig. 2. Figure by which we introduce the length of the section in the integral.

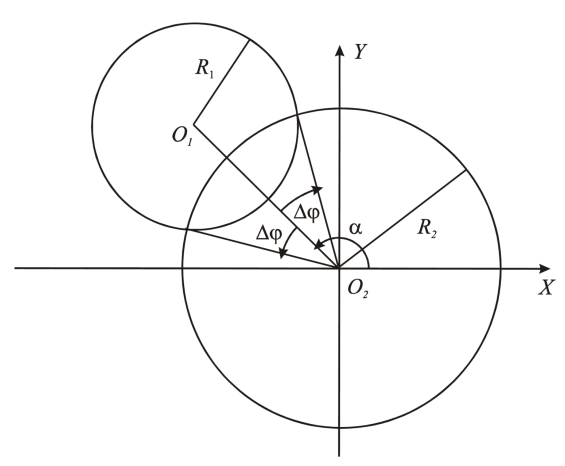

Fig. 3. Figure by which we determine limits of the integral.

Half of the angle under which two intersection points are seen, viewed from the centre of the other circle, is

$$
\Delta \varphi\left(R_{1}, R_{2}, X\right)=\pi-\operatorname{mfarccos}\left(\frac{R_{1}^{2}-R_{2}^{2}-X^{2}}{2 X R_{2}}\right) .
$$

In the case of their cutting, we can calculate half of the angle under which two intersection spots are seen, viewed from the centre of the other circle. It is given by:

$$
\Delta \varphi\left(R_{1}, R_{2}, X\right)=\pi-\arccos \left(\frac{R_{1}^{2}-R_{2}^{2}-X^{2}}{2 X R_{2}}\right) .
$$

If the circles do not intersect, then the function $\arccos (x)$ is zero.

Let us determine the length of the intersection of two intervals $(a, b)$ and $(c, d)$. It can be noted in the form:

$$
D(a, b, c, d)=\max [0, \min (b, d)-\max (a, c)] .
$$

Now we can solve the integral of $\varphi_{m}$ in (9).

Let us consider the deviation, for example, as in Fig. 2. The circle whose centre is on the edge of the disk is set up under the angle $\alpha$ and its radius is $R_{E}$. In Fig. 2, the centre of the Descartes's coordinate system $(x=0, y=0, z=0)$ is in the point $O$. We calculate the value $A$ in the point $(x, y, z)$. Its projection on the plane $x O y$ is in the point $O_{m}$ which is the centre of the cylindrical coordinate system given by (8). Let us determine the angle under which the illuminated part of the disk $I_{\varphi_{m}}$ is seen. From the size of the interval of the angles under which the whole disk is seen we take up the size of the intersection of the interval and the one under which the cut part is seen. In Fig. 2, the circular arc which covers the illuminated part of the circular disk goes from $A$ to $B$, opposite to the direction clockwise and it is seen under the angle $\varphi_{b} \max -\varphi_{b} \min$.

The cut part which causes the deviation goes from $C$ to $D$ and it is seen under the angle $\varphi_{d \max }-\varphi_{d \min }$. So:

$$
\begin{aligned}
& I_{\varphi_{m}}=\varphi_{b \max }-\varphi_{b \min } \\
& \quad-D\left(\varphi_{b \min }, \varphi_{b \max }, \varphi_{d \min }, \varphi_{d \max }\right),
\end{aligned}
$$

where $\varphi_{b \min }=\varphi_{b}-\Delta \varphi_{b}, \quad \varphi_{b \max }=\varphi_{b}+\Delta \varphi_{b}$, $\varphi_{d \min }=\varphi_{d}-\Delta \varphi_{d}$, and $\varphi_{d \max }=\varphi_{d}+\Delta \varphi_{d}$.

The coordinates of the points $O$ and $O_{m}$, observed from the coordinate origin $O_{m}$, are: $O(-x,-y)$ and $O_{E}(-x+R \cos (\alpha),-y+R \sin (\alpha))$. The determined straight lines $\overline{O O_{m}}$ and $\overline{O O_{E}}$ divide in half the angles $\angle A O_{m} B$ and $\angle C O_{m} B$, respectively. For this reason,

$$
\begin{aligned}
& \varphi_{b}=\arctan \left(\frac{-y}{-x}\right), \\
& \varphi_{d}=\arctan \left(\frac{-y+R \sin (\alpha)}{-x+R \cos (\alpha)}\right) .
\end{aligned}
$$

where the function arctan is in the interval $(-\pi, \pi]$, and, importantly, takes into account in which quadrant there are the points $O$ and $O_{E}$. Using (13), we get:

$$
\begin{aligned}
& \Delta \varphi_{b}=\Delta \varphi\left(R, \rho_{m}, \sqrt{x^{2}+y^{2}}\right) \\
& \Delta \varphi_{d}= \\
& \Delta \varphi\left(R_{E}, \rho_{m}, \sqrt{(-x+R \cos (\alpha))^{2}+(-y+R \sin (\alpha))^{2}}\right) .
\end{aligned}
$$

In this way, we can express (9) as:

$$
I_{\varphi_{m}}=\phi\left(\rho_{m}\right)
$$

where $\phi\left(\rho_{m}\right)$ is obtained when substituting (16) and (17) into (15). Note that $A_{h}$ in (9) depends only on $\rho_{m}$ and $z$, therefore $(7)$ can be written as

$$
\begin{aligned}
& A_{h}(x, y, z)=-\frac{\mathrm{i} A_{0}}{\lambda} \int_{0}^{\sqrt{x^{2}+y^{2}}+R} \phi\left(\rho_{m}\right) \frac{\mathrm{e}^{\mathrm{i} k r}}{r} \\
& \times\left(1-\frac{1}{\mathrm{i} k r}\right) \cos (\theta) \mathrm{d} \rho_{m},
\end{aligned}
$$

where $r$ and $\theta$ depend on $\rho_{m}$ by relation (8).

The expression (5) can be written in the form suitable for numerical calculation. The relative intensity of Poisson's spot, i.e.,

$$
\frac{i}{i_{0}}=\frac{|A|^{2}}{\left|A_{0}\right|^{2}}
$$

can now be calculated numerically for the arbitrarily chosen $z$, using an algorithm with the parameters $R, R_{E}, \alpha$ and $\lambda$. We can change $R_{E}$ and $\alpha$ 
both by the value and the number for given $R, z$ and $\lambda$. The form of circular deviations depends on that. For given $R, R_{E}, \alpha$ and $\lambda$ the dependence of the relative intensity from the distance $z$ from the screen can be examined, too. Let us note that by this method both the symmetrical and the asymmetrical circular deviations can be observed both in $2 \mathrm{D}$ and $3 \mathrm{D}$ by the suitably written algorithms.

\subsection{Algorithm}

On the triangle $\Delta O_{1} O_{2} A$ (Fig. 3) we apply the cosine theorem $\left(\mathrm{O}_{1} \mathrm{O}_{2}=\mathrm{X}\right)$ :

$$
\begin{aligned}
& R_{1}^{2}=R_{2}^{2}+X^{2}-2 R_{2} X \cos (\Delta \phi), \\
& \cos \Delta \phi=\frac{R_{1}^{2}-R_{2}^{2}-X^{2}}{-2 X R_{2}} \\
& \Delta \phi=\arccos \left(-\frac{R_{1}^{2}-R_{2}^{2}-X^{2}}{2 X R_{2}}\right), \\
& \Delta \phi=\pi-\arccos \left(\frac{R_{1}^{2}-R_{2}^{2}-X^{2}}{2 X R_{2}}\right) .
\end{aligned}
$$

\subsection{Numerical evaluation}

Calculation of $A(x, y, z)$ for given parameters $R$, $R_{E}, \lambda$, and for an array of angles $\alpha_{i}$ which indicates the position of counts, requires the following steps of numerical evaluations:

- define the function, that for $O_{1 x}, O_{1 y}, R_{1}$, and $R_{2}$ determines an interval $(\alpha-\Delta \phi, \alpha+\Delta \phi)$, where $\alpha=\arctan \left(\frac{O_{1 x}}{O_{1 y}}\right)$, and $\Delta \phi$ is given by (12) with $X=\sqrt{O_{1 x}^{2}+O_{1 y}^{2}}$,

- calculate the integral (19),

- since for each cutting out $\rho_{m}$ is fixed, one can calculate an interval from (1), where:

$$
\begin{aligned}
& O_{1 x_{i}}=-x+R \cos \left(\alpha_{i}\right), \\
& O_{1 y_{i}}=-y+R \cos \left(\alpha_{i}\right), \\
& R_{1}=R_{E}, \quad R_{2}=\rho_{m} .
\end{aligned}
$$

We denote this interval by $\left(\phi_{n \min }, \phi_{n \max }\right)$.

- determine:

$$
\begin{aligned}
& I_{\phi_{m}}=\phi_{\max }-\phi_{\min } \\
& -\sum_{n} D\left(\phi_{\min }, \phi_{\max }, \phi_{n \min ,}, \phi_{n \max }\right) .
\end{aligned}
$$

- calculate (5).

\section{Influence of larger deviations on Poisson's spot}

Let us apply the described method to the configurations with larger deviations from the ideal circular cross-section. We simulate the deviations by
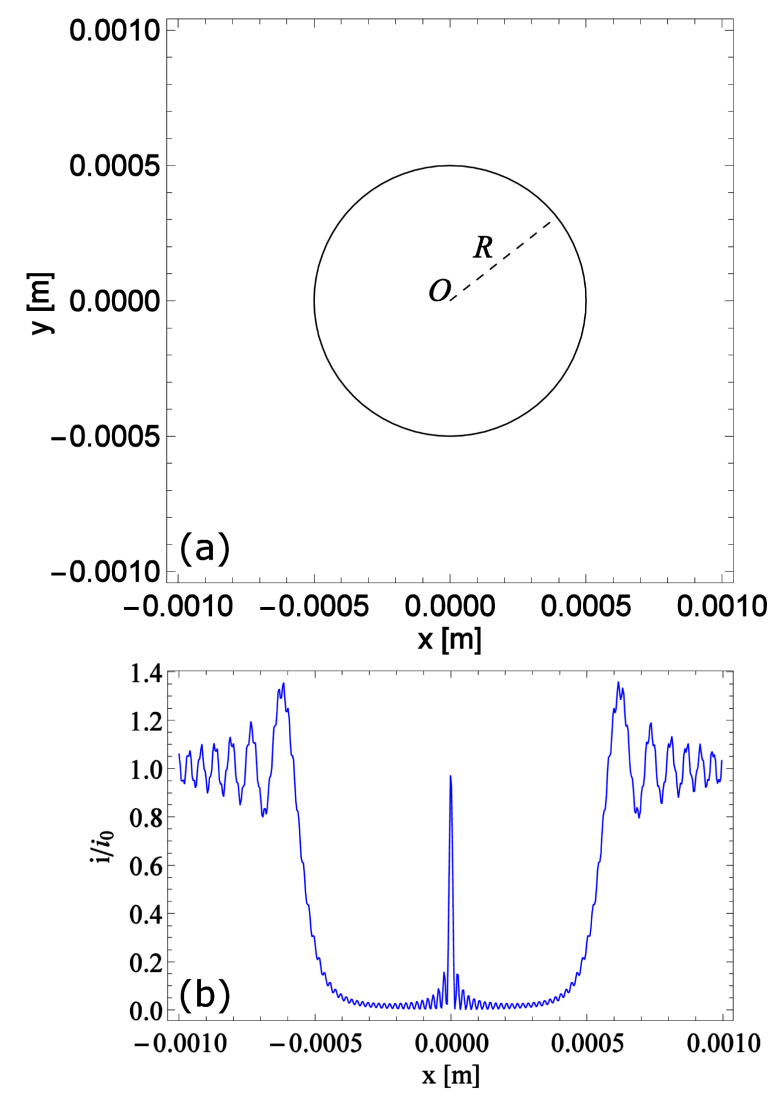

Fig. 4. (a) Cross-section of the circular object of the radius $R=0.5 \mathrm{~mm}$. (b) The relative intensity of monochromatic light on the distance $z=0.04 \mathrm{~m}$ behind the circular object depending on $x$.

cutting larger parts from the circular cross-section by the circles whose centres are on the edge of the object. The radius of the object is $R=0.5 \mathrm{~mm}$ and it is lightened by a monochromatic laser light of the wavelength $\lambda=500 \mathrm{~nm}$. Without the deviations, the relative intensity of Poisson's spot depending on $x$ on the distance $z=0.04 \mathrm{~m}$ behind the disk is shown in Fig. 4.

Now, from the circular cross-section we cut one part by the circle of the radius $R_{E}=0.2 \mathrm{~mm}$ whose centre is on the edge of the disk in the point $(x, y)=(R, 0),(\alpha=0)$. We shall get the configuration that is shown in Fig. 5. We model the experiment in which the object of such a cross-section is lightened by the laser light of $\lambda=500 \mathrm{~nm}$.

In the same distance behind the lightened object, we shall get a diffractional picture that also contains a bright spot in the centre of the shadow (Fig. 6a and b). The relative intensity of the bright spot lessened but it keeps the shape of Poisson's spot. In this case, the relative intensity in the centre of the shadow is $i / i_{0} \approx 0.82$.

The dependence of the relative intensity of Poisson's spot from the size of the deviation, from the distance $z$ from the object and from the position of the deviation on the cross-section of the object is shown in Fig. 7. One can see that the dependence of 

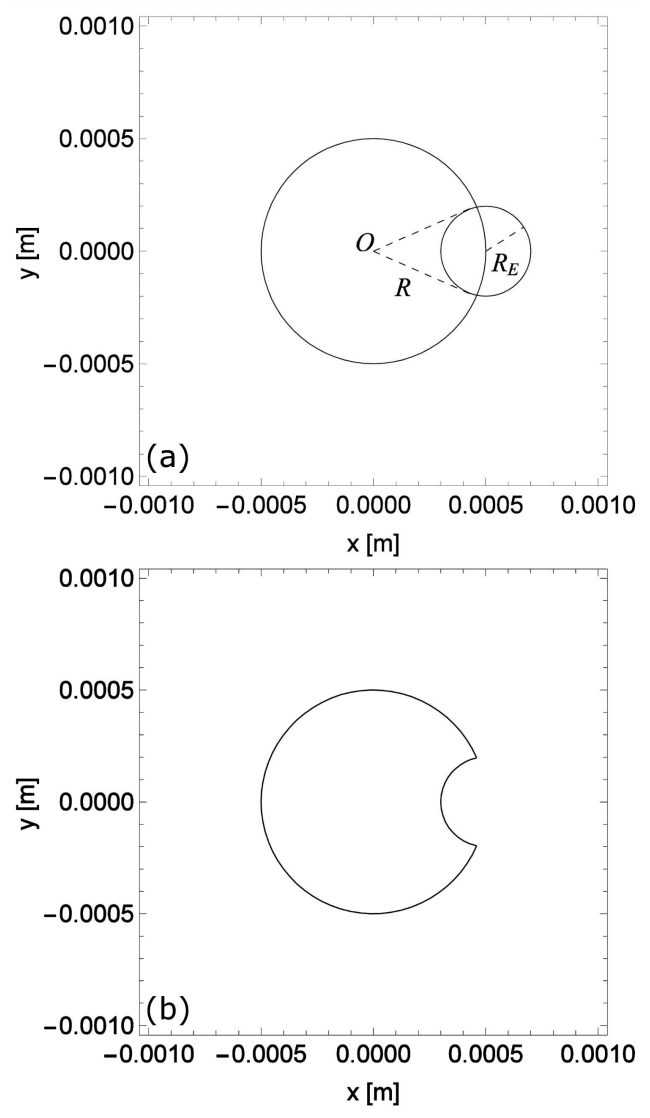

Fig. 5. (a) The scheme of the getting of the one larger deviation of the radius $R_{E}=0.2 \mathrm{~mm}$ and with the position $\alpha=0$ on the circle of the radius $R=0.5 \mathrm{~mm}$. (b) The cross-section of the object with one larger deviation.

the relative intensity from the size of the damage of the disk is of an oscillating kind. The Fresnel zone concept gives a very physical explanation. When the disk is not damaged, the only contribution to the relative intensity of Poisson's spot is given by the first Fresnel zone above the edge of the disk [12]. The cut part unblocks a certain number of Fresnel zones which were unblocked by the disk. They decrease or increase the relative intensity of Poisson's spot in regard to the previous value. In fact, that depends on the number and extension of the zones which are partially unblocked, and on their surfaces. Therefore, with increasing $R_{E}$, the relative intensity falls although not continuously. If the distance of the source of light from the disk is much longer than the distance $z$ on which the diffraction picture is observed, then the radius of $n^{\text {th }}$ zone can be estimated as $r_{n} \approx \sqrt{n \lambda z}$ [19]. The number and the surfaces of the unblocked zones are easily determined. The widths of the neighbouring zones are given by $\Delta r_{n} \approx r_{n}-r_{n-1}=r_{n}-\sqrt{r_{n}^{2}-\lambda z}$. Note that the width of the zone is increased from the edge towards the centre of the disk. Their surfaces in the unblocked part are approximately equal. The secondary waves which start from the neighbouring zones do not cross the same paths towards

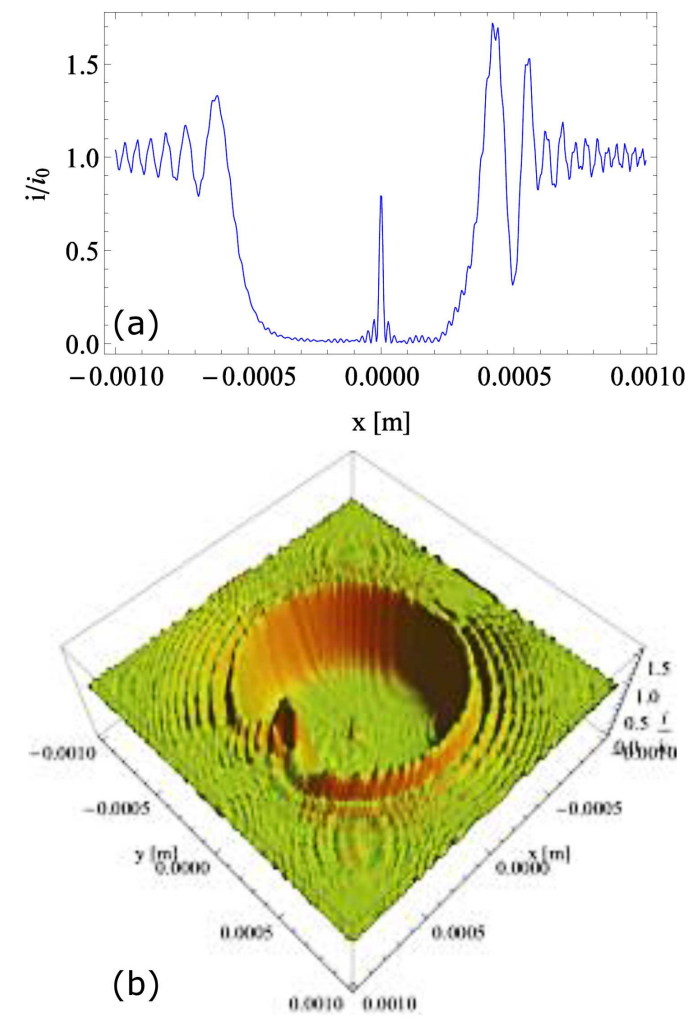

Fig. 6. (a) The relative intensity of the monochromatic light behind the object shown in Fig. 5, (a) in $2 \mathrm{D}$ and (b) in $3 \mathrm{D}$.
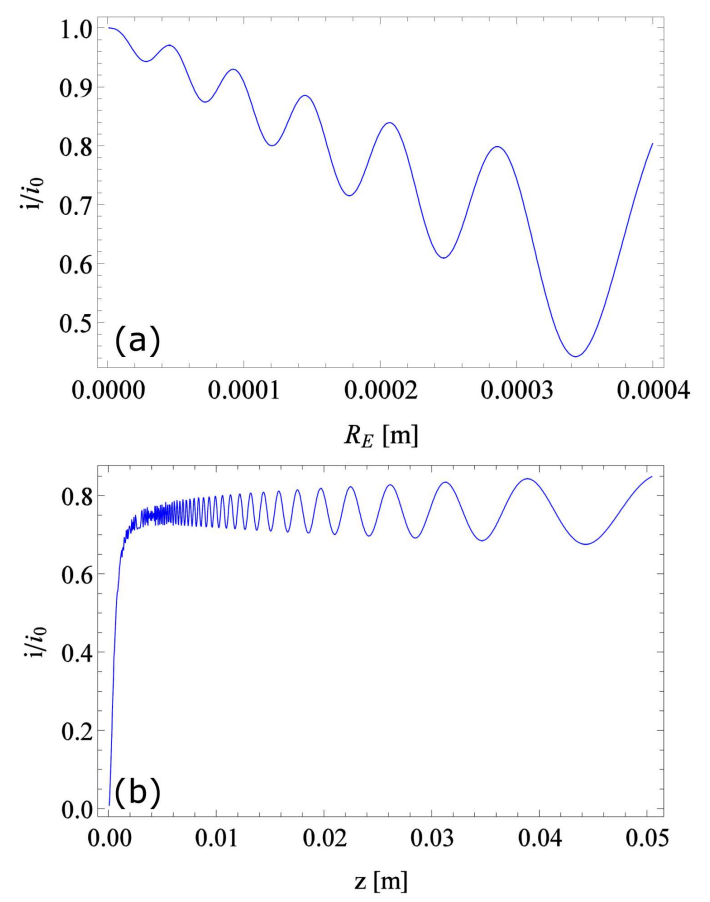

Fig. 7. (a) The oscillatory dependence of the relative intensity of Poisson's spot from the size of the deviation (from $R_{E}$ ) for $z=0.04 \mathrm{~m}$. (b) The oscillatory dependence of the relative intensity of Poisson's spot from the distance $z$ from the lightened object for $R_{E}=0.2 \mathrm{~mm}$. 

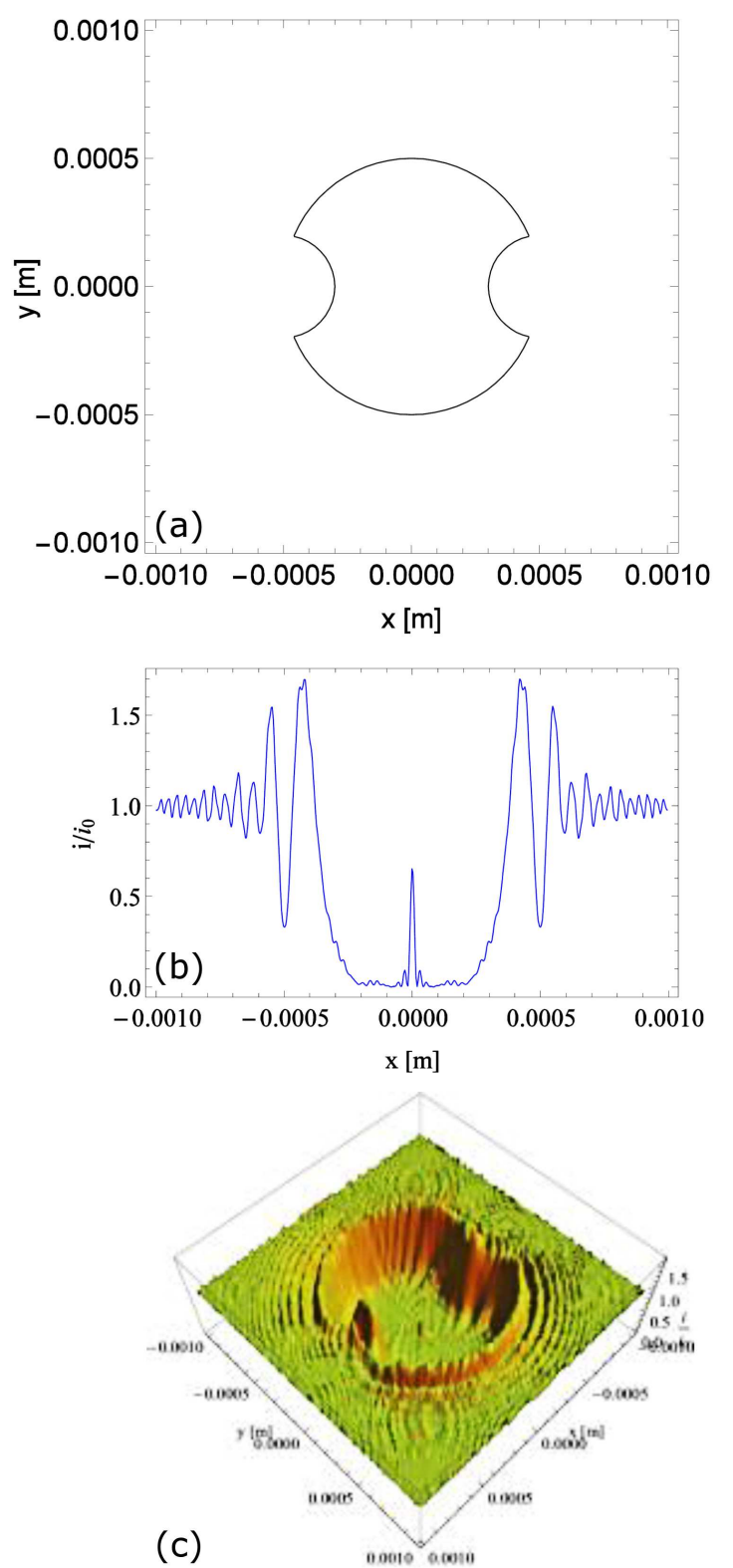

Fig. 8. (a) The cross-section of the object when from the circular disk of the radius $R=0.5 \mathrm{~mm}$ we cut two parts by the circles of the same radii $R_{E}=0.2 \mathrm{~mm}$ with the positions $\alpha_{1}=0$ and $\alpha_{2}=$ $\pi$. (b) The relative intensity of monochromatic light behind the object depending on $x$ on the distance $z=0.04 \mathrm{~m}$ in $2 \mathrm{D}$ and $\mathrm{c}$ ) in $3 \mathrm{D}$.

the central spot on the distance $z=0.04 \mathrm{~m}$. They rather arrive with different phases at it. The waves that start from the even zones are in the phase and the waves that start from the odd zones are also in the phase. The waves from the even and odd zones are in the opposite phases. Because of that, the relative intensity of the central bright spot falls oscillatorily. In the case when $R_{E}=0.2 \mathrm{~mm}$, seven Fresnel zones are unblocked, thus the curve seen in Fig. 7a contains 4 local minima and 3 local maxima. Such a behaviour of a relative intensity with increasing $R_{E}$ is expected.
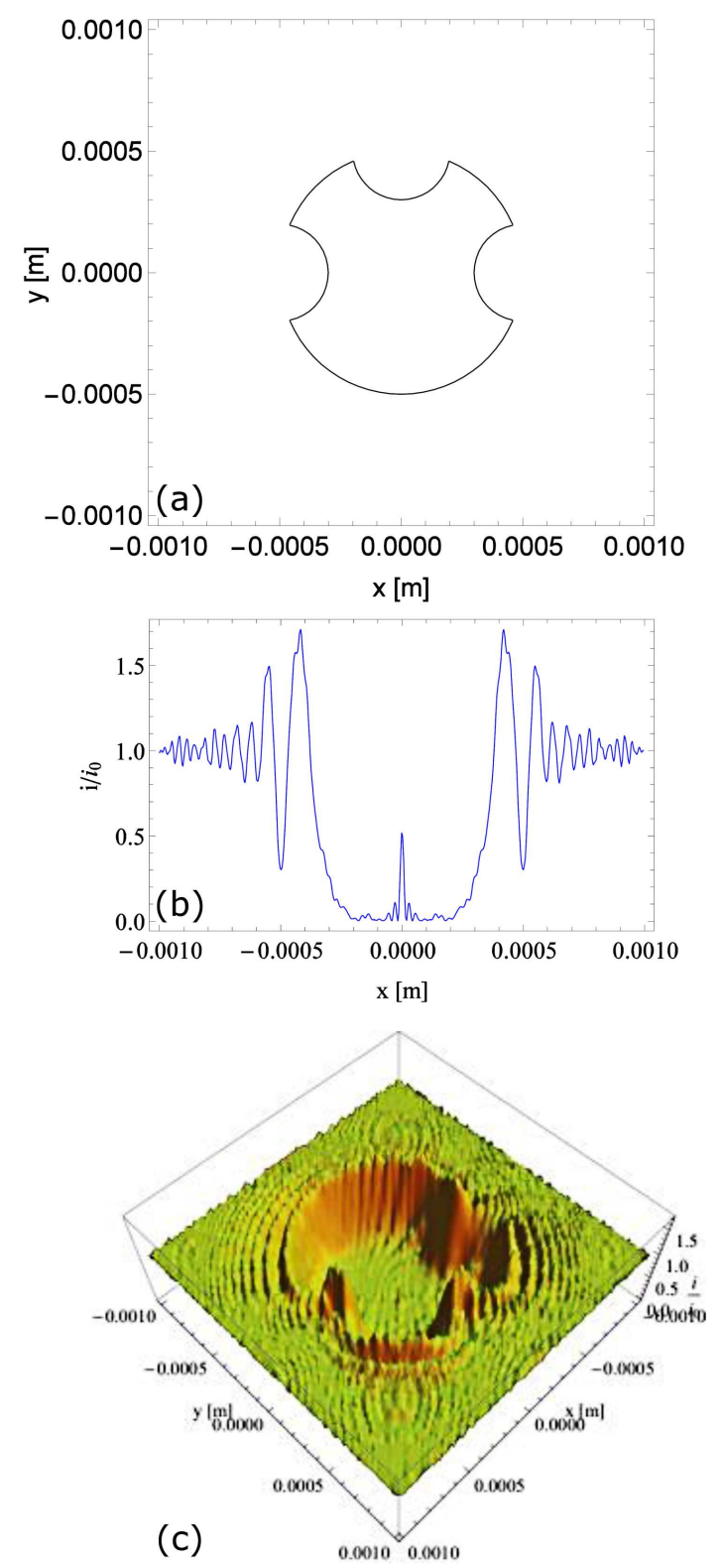

Fig. 9. (a) The cross-section of the object when from the circular disk of the radius $R=0.5 \mathrm{~mm}$ we cut three parts by the circles of the same radii $R_{E}=0.2 \mathrm{~mm}$ with the position $\alpha_{1}=0, \alpha_{2}=\pi$ and $\alpha_{3}=\frac{\pi}{2}$. (b) The relative intensity of monochromatic light behind the object depending on $x$ on the distance $z=0.04 \mathrm{~m}$ in $2 \mathrm{D}$ and (c) in 3D.

Figure 7b shows that the dependence of the relative intensity of the central bright spot from $z$ is also oscillatory. In this case, when $z$ is increased, the radius of the Fresnel zones within the damage grows, while the number of the zones in the damage falls. Therefore, we have the relative intensity's increase and decrease in regard to the previous. The relative intensity will not fall continuously. It will oscillate around $i / i_{0} \approx 0.75$. When $z$ is further increased, the number of the maxima and minima decrease until the central bright spot is lost. When the width of the first Fresnel zone is beneath 
the edge of the disk $\Delta r \approx R_{E}$, Poisson's spot is almost lost. This happens for $z \approx 0.32 \mathrm{~m}$ (based on the expression for width of neighbouring Fresnel zones, when $r_{n}=R$ and $\left.\Delta r_{n}=R_{E}\right)$. Moreover, the damage contains only one Fresnel zone. In case when no damage occurs, the dependence of relative intensity on $z$ goes much faster towards $i / i_{0}=1$ and does not oscillate. For large values of $z$, the dependence behaves like an asymptote. By the damage, the dependence of the relative intensity on $z$ oscillates around a line without inclination, which is below $i / i_{0}=1$.

The procedure of simulation for two, three and a larger number of deviations is similar as in the case of one deviation. The physical explanation is qualitatively the same. The appropriate dependence differs only quantitatively. Figures 8 and 9 show the appropriate dependences of the relative intensity of Poisson's spot for two and three deviations from the ideal circular cross-section, respectively. In the case of two deviations $i / i_{0} \approx 0.67$ in the centre of the shadow, while in the case of three deviations $i / i_{0} \approx 0.53$.

\section{Impact of "circular rougness" on the relative intensity of Poisson's spot}

In paper [12], the roughness is simulated by adding deviations to the first Fresnel zone above the disk. We simulated the roughness by the damage of the disk by the semi-circular deviations in the first Fresnel zone beneath the edge of the disk. Because of that, we named it "the circular roughness". Let us consider how such roughness of the circular opaque disk simulated by 48 circles of the same radii influences the intensity of Poisson's spot (Fig. 10). The centres of the circles are on the edge of the disk and they cut equal parts from the observed disk. Let the monochromatic light of wavelength $\lambda=500 \mathrm{~nm}$ fall on such a rough circular opaque disk of radius $R=0.5 \mathrm{~mm}$.

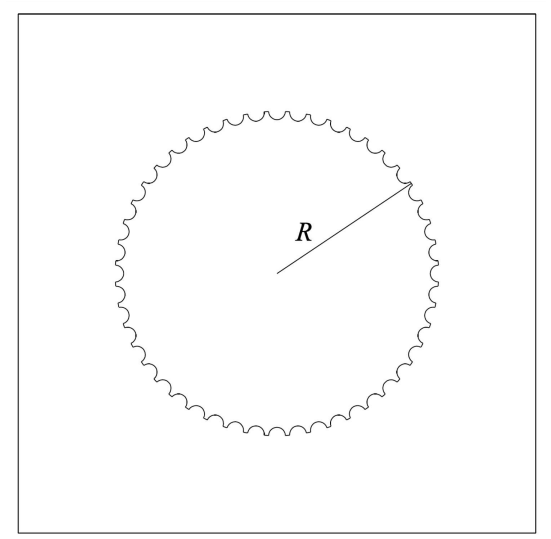

Fig. 10. The simulation of the circular opaque disk roughness of the radius $R=0.5 \mathrm{~mm}$ with 48 circles of the same radii with the centres on the edge of the disk.

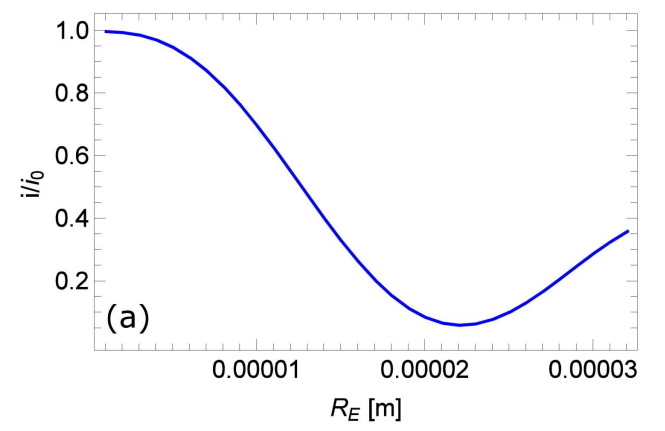

(b)

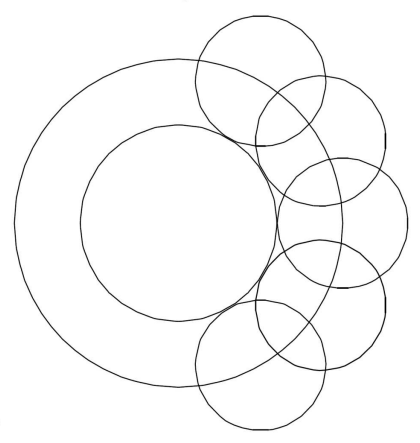

Fig. 11. (a) The dependence of the relative intensity of Poisson's spot from $R_{E}$ for $z=0.04 \mathrm{~m}$ behind the rough disk. (b) Overlap of cuttings for $R_{E}>20 \mu \mathrm{m}$.

Let such a disk be situated in the plane $z=0$, with the centre in the coordinate origin. Using the described method, we watched the relative intensity of Poisson's spot behind the disk in the distance $z=0.04 \mathrm{~m}$ from $x O y$ plane.

We were examining the dependence of the relative intensity of Poisson's spot for the different radii of the circles $R_{E}$ (Fig. 11a). All the values $R_{E}$ there are in the first Fresnel zone beneath the edge of the disk. The width of that zone is $\Delta r \approx 20 \mu \mathrm{m}$. The relative intensity grows with the increase of $R_{E}$ as the roughness falls effectively because of the overlap of the cuttings (Fig. 11b).

To show how the size of the cut parts influences the relative intensity of Poisson's spot, we chose three values of $R_{E}=2 \mu \mathrm{m}, R_{E}=10 \mu \mathrm{m}$ and $R_{E}=20 \mu \mathrm{m}$. At the same time, we examined the dependence of the relative intensity of Poisson's spot from $z$ for these chosen values of $R_{E}$ (Figs. 12-14).

By this method, the light distribution of the relative intensity behind the rough disk (in all three cases) may be displayed in 3D (Figs. 15-17).

\section{Discussion}

The method presented in Sect. 2 refers to the semi-circular shape of the damage of the circular object. The edge of the damaged disk is ideally smooth. When introducing large damages of the circular opaque disk (Sect. 3), Poisson's spot is not lost. In fact, its relative intensity reveals an unexpected property. It does not fall continuously yet 

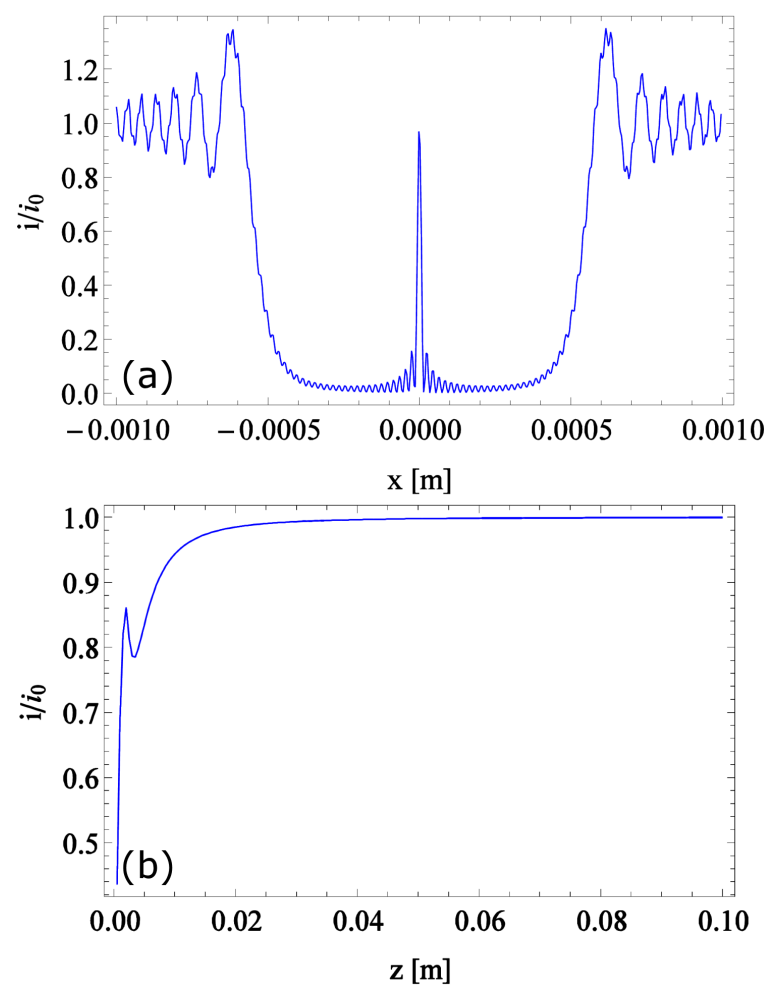

Fig. 12. (a) The relative intensity of monochromatic light behind the object depending on $x$ for $R_{E}=2 \mu \mathrm{m}$, on the distance $z=0.04 \mathrm{~m}$. (b) The relative intensity of Poisson's spot depending on $z$.
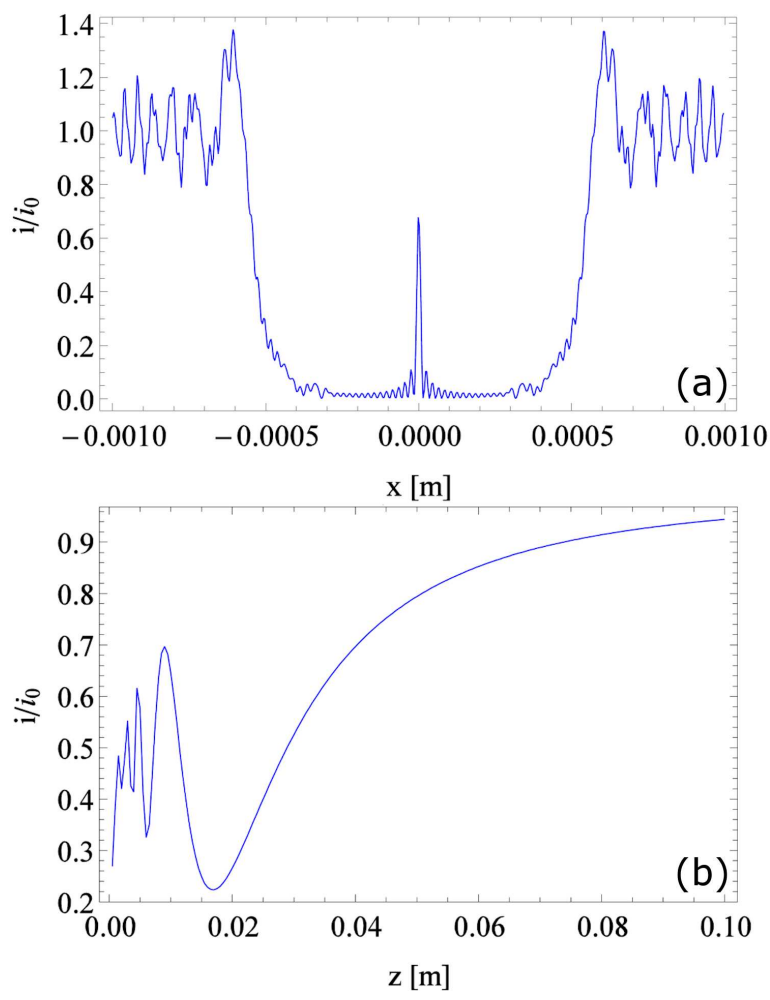

Fig. 13. (a) The relative intensity of monochromatic light behind the object depending on $x$ for $R_{E}=10 \mu \mathrm{m}$ on the distance $z=0.04 \mathrm{~m}$. (b) The relative intensity of Poisson's spot depending on $z$.
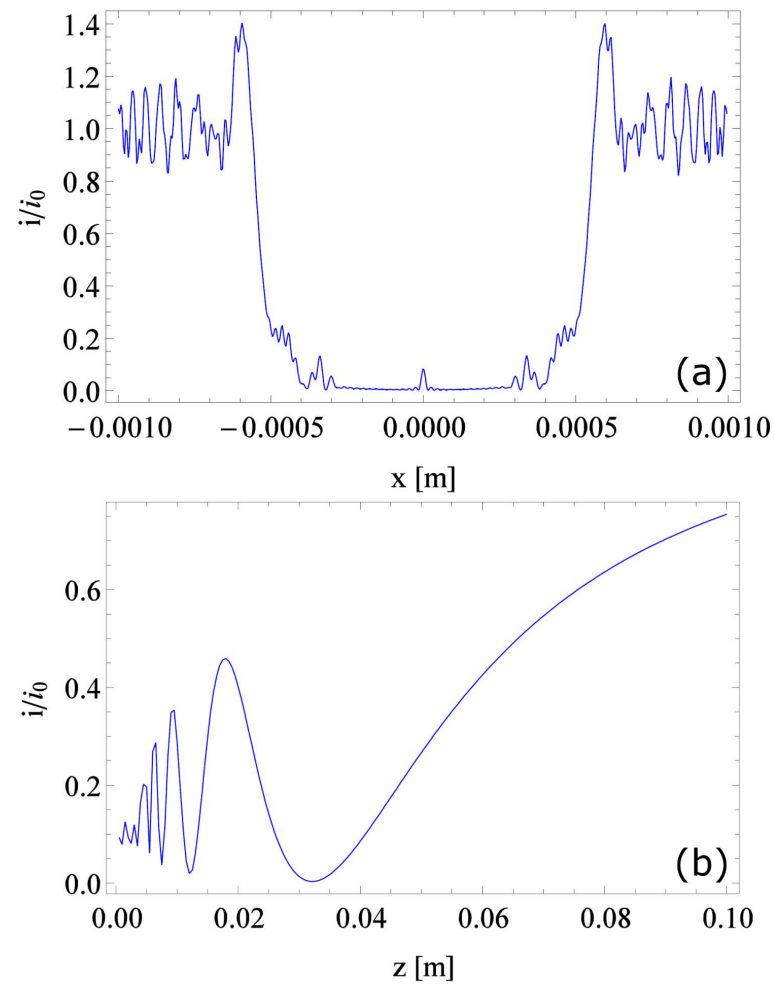

Fig. 14. (a) The relative intensity of monochromatic light behind the object depending on $x$ for $R_{E}=20 \mu \mathrm{m}$ on the distance $z=0.04 \mathrm{~m}$. (b) The relative intensity of Poisson's spot depending on $z$.
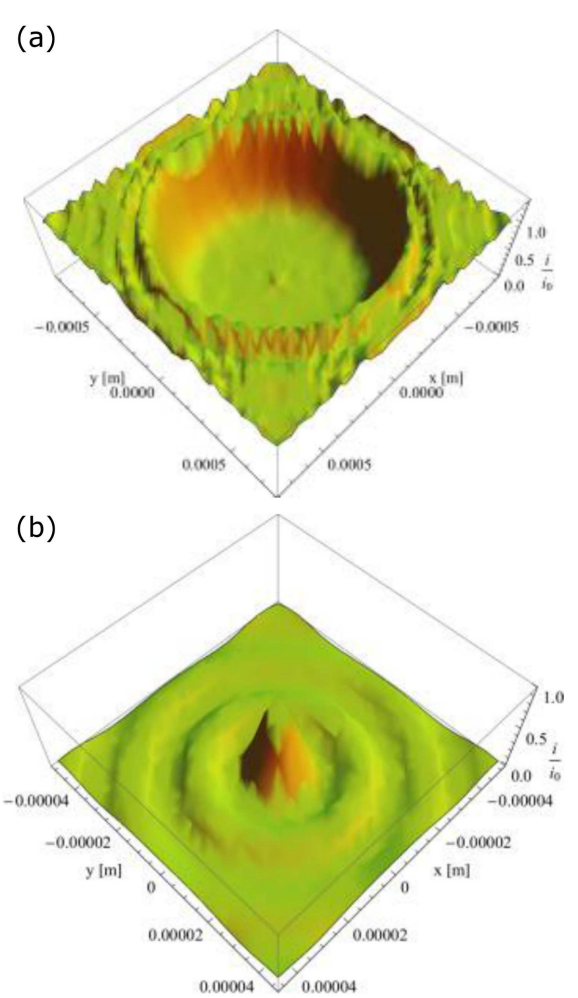

Fig. 15. (a) The relative intensity of monochromatic light behind the object for $R_{E}=2 \mu \mathrm{m}$, on the distance $z=0.04 \mathrm{~m}$ in 3D. (b) The increased lightened part around the centre of the shadow on (a). 


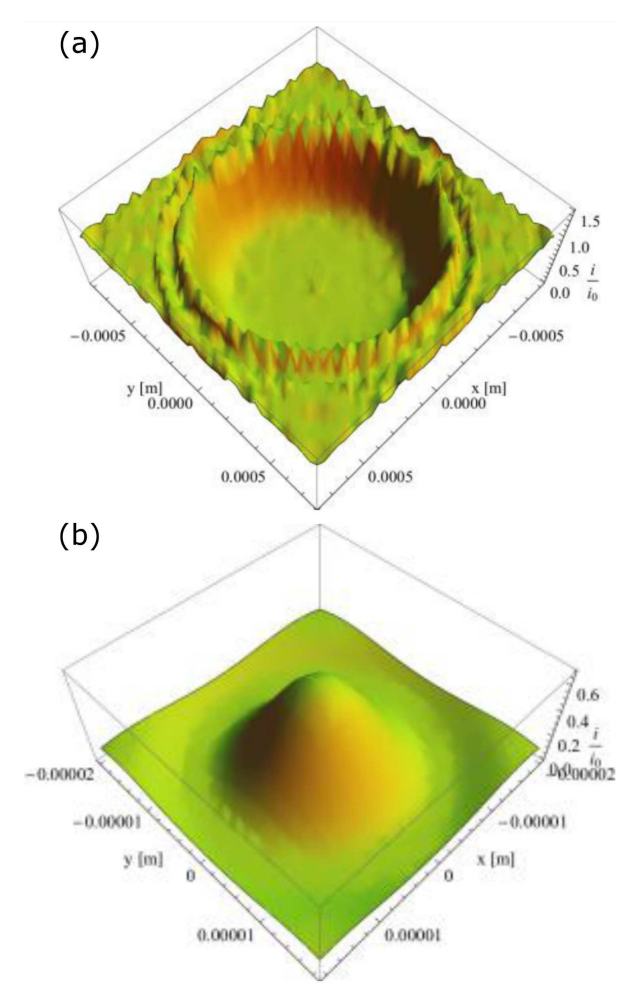

Fig. 16. (a) The relative intensity of monochromatic light behind the object for $R_{E}=10 \mu \mathrm{m}$ on the distance $z=0.04 \mathrm{~m}$ in $3 \mathrm{D}$. (b) The increased lightened part around the centre of the shadow on (a).

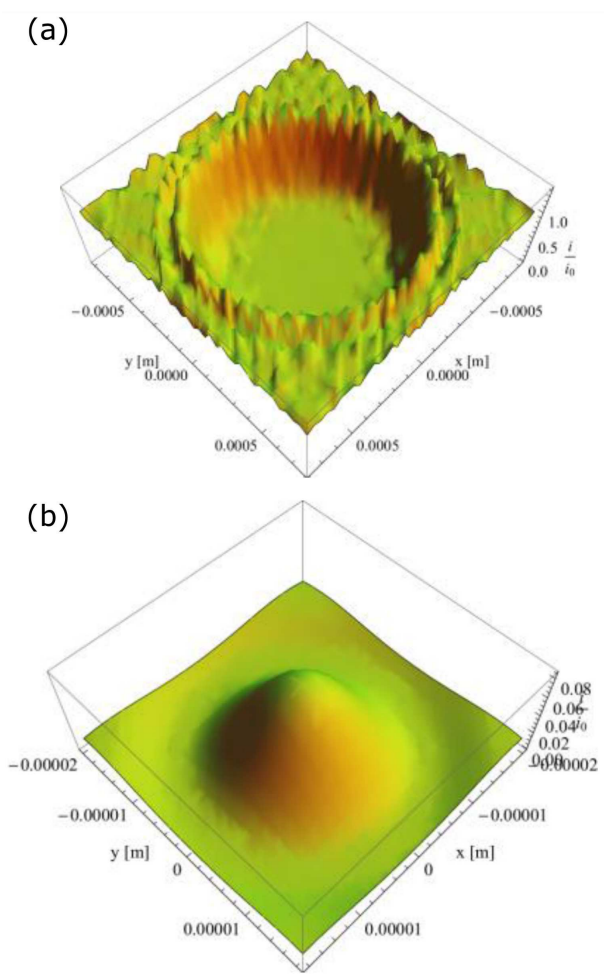

Fig. 17. (a) The relative intensity of monochromatic light behind the object for $R_{E}=20 \mu \mathrm{m}$ on the distance $z=0.04 \mathrm{~min} 3 \mathrm{D}$. (b) The increased lightened part around the centre of the shadow which cannot be seen on (a). oscillatorily, which is explained by the concept of the Fresnel zones. It can be shown that Poisson's spot will exist while the damage does not completely unblock the first Fresnel zone around the centre of the disk. The dependence of the relative intensity of Poisson's spot from the distance $z$ of the disk has also displayed oscillatory behaviour. The relative intensity falls and grows oscillatorily around a definite value which depends on the size of the damage. It is shown that the relative intensity of Poisson's spot does not depend on the angular position of the damage, regardless of the number of damages. However, the diffraction image behind the disk changes qualitatively. The dependence of the relative intensity of Poisson's spot behind the damaged circular disk on the wavelength of the light was not subject of this research.

A differently shaped surface roughness of the circular disk can also be simulated by the method presented in Sect. 4, in the way it was done in [12]. In fact, the obtained dependence of the relative intensity of Poisson's spot on surface roughness is similar to the results reported in [12]. Our results in Sect. 4 show that even small deviations from the ideal circular cross-section lead to the reduction of the relative intensity of Poisson's spot. When $R_{E}=10 \%$ from $\Delta r$ (see Figs. 12a and 15) the reduction is slight, but when $R_{E}=50 \%$ from $\Delta r$ (see Figs. 13a and 16), then the reduction is about $30 \%$. When $R_{E} \approx \Delta r$, then Poisson's spot is almost lost (see Figs. 14a and 17). The reduction in intensity also depends on the chosen wavelength and distance $z$.

\section{Conclusion}

The described method and results offer new possibilities for experimental and theoretical research on the properties of Poisson's spot behind a damaged disk. For example, the examining of the influence of larger deviations on Poisson's spot can be applied in theoretical and experimental researches of red blood cell damages for diagnosis of anemia. It particularly refers to the diagnosis of sickle cell anemia. A cross-section of a healthy red blood cell is approximate to the ideal circular disk $(R \approx 6.9 \mu \mathrm{m})$. This method gives new possibilities to research on how the roughness influences the relative intensity of Poisson's spot in the experiments both with light and particles with non-zero rest-mass. The introduced functions and the integrals enable the examination of the field of photons and EME flow lines behind the damaged disk. It will be the subject of future research.

\section{Acknowledgments}

Author (D.A.) acknowledges financial support from the grant No. III45016 of the Serbian Ministry of Education, Science and Technological Development. 


\section{References}

[1] R.L. Lucke, Eur. J. Phys. 27, 193 (2006).

[2] J.E. Harvey, J.L. Forgham, Am. J. Phys. 52, 243 (1984).

[3] A. Fresnel, Oeuvres Compètes, Imprimerie Impériale, Paris 1866.

[4] I. Newton, Opticks: Or, A Treatise of the Reflections, Refractions, Inflections and Colours of Light, Royal Society, London 1704.

[5] M. Davidović, A.S. Sanz, M. Božić, D. Arsenović, D. Dimić, Phys. Scr. T153, 014015 (2013).

[6] A.S. Sanz, M. Davidović, M. Božić, S. Miret Artés, Ann. Phys. 325, 763 (2010).

[7] M. Davidović, A.S. Sanz, D. Arsenović, M. Božić, S. Miret-Artes, Phys. Scr. T135, 014009 (2009).

[8] D. Arsenovic, D. Dimic, M. Bozic, Phys. Scr. T162, 014039 (2014).

[9] M. Gondran, A. Gondran,Am. J. Phys. 78, 598 (2010).
[10] A. Sommerfeld, Ann. Phys. 18, 663 (1883).

[11] Lord Rayleigh, Philos. Mag. 43, 259 (1897).

[12] T. Reisinger, A.A. Patel, H. Reingruber, K. Fladischer, W.E. Ernst, G. Bracco, H.I. Smith, B. Holst, Phys. Rev. A 79, 053823 (2009).

[13] T. Reisinger, G. Bracco, B. Holst, New J. Phys. 13, 065016 (2011).

[14] E.D. Dauger, Comput. Phys. 10, 591 (1996).

[15] S. Shiri, W. Wasylkiwskyj, J. Opt. 15 , 035705 (2013).

[16] T. Reisinger, P.M Leufke, H. Gleiter, H. Han, New J. Phys. 19, 033022 (2017).

[17] G.L. Abramyan, Radiophys. Quantum Electron. 24, 132 (1981).

[18] S. Ganci, Am. J. Phys. 73, 83 (2005).

[19] A. Sommerfeld, Lectures on Theoretical Physics, Vol. IV, Academic Press Inc., New York 1954. 